\title{
Single-locus complementary sex determination absent in Heterospilus prosopidis (Hymenoptera: Braconidae)
}

\author{
Z Wu ${ }^{1}$, KR Hopper ${ }^{2}$, PJ Ode ${ }^{2,4}$, RW Fuester ${ }^{2}$, M Tuda $^{3}$ and GE Heimpel ${ }^{1}$ \\ ${ }^{1}$ Department of Entomology, University of Minnesota, St Paul, MN, USA; ${ }^{2} U S D A$, ARS, Beneficial Insects Introduction Research \\ Laboratory, Newark, DE, USA; ${ }^{3}$ Institute of Biological Control, Faculty of Agriculture, Kyushu University, Fukuoka, 812-8581 Japan
}

In the haplodiploid Hymenoptera, haploid males arise from unfertilized eggs, receiving a single set of maternal chromosomes while diploid females arise from fertilized eggs and receive both maternal and paternal chromosomes. Under single-locus complementary sex determination (sl-CSD), sex is determined by multiple alleles at a single locus. Sex locus heterozygotes develop as females, while hemizygous and homozygous eggs develop as haploid and diploid males, respectively. Diploid males, which are inviable or sterile in almost all cases studied, are therefore produced in high frequency under inbreeding or in populations with low sex allele diversity. CSD is considered to be the ancestral form of sex determination within the Hymenoptera because members of the most basal taxa have CSD while some of the more derived groups have other mechanisms of sex determination that produce the haplo-diploid pattern without penalizing inbreeding. In this study, we investigated sex determination in Heterospilus prosopidis Viereck, a parasitoid from a relatively primitive subfamily of the Braconidae, a hymenopteran family having species with and without CSD. By comparing sex ratio and mortality patterns produced by inbred and outbred females, we were able to rule out sl-CSD as a sex determination mechanism in this species. The absence of $s$-CSD in $H$. prosopidis was unexpected given its basal phylogenetic position in the Braconidae. This and other recent studies suggest that sex determination systems in the Hymenoptera may be evolutionary labile.

Heredity (2005) 95, 228-234. doi:10.1038/sj.hdy.6800720; published online 3 August 2005

Keywords: Hymenoptera; Braconidae; sex determination; haplo-diploidy; complementary sex determination; Heterospilus

\section{Introduction}

In members of the Hymenoptera that reproduce sexually, unfertilized eggs develop as haploid males, and fertilized eggs develop as diploids that are typically females. This mode of sex determination is known as haplodiploidy. The genetic mechanisms underlying haplodiploidy remain poorly understood, but in some hymenopterans, haplo-diploidy can be attributed to a single, highly polymorphic sex locus. Under single-locus complementary sex determination (sl-CSD), eggs that are heterozygous at the sex locus develop as females while hemizygous and homozygous eggs develop as haploid and diploid males, respectively (Whiting, 1943; Beye et al, 2003). Diploid males may be inviable, sterile, or functionally reproductive (Stouthamer et al, 1992; Cook and Crozier, 1995; Cowan and Stahlhut, 2004), and they are produced under inbreeding or in populations with low sex allele diversity. While sterile or inviable diploid males constitute a genetic load associated with CSD (Stouthamer et al, 1992; Werren, 1993; Cook and Crozier, 1995), the recent finding of functionally reproductive

Correspondence: GE Heimpel, Department of Entomology, University of Minnesota, 1980 Folwell Ave., St Paul, MN 55108, USA.

E-mail: heimp001@umn.edu

${ }^{4}$ Current address: Department of Entomology, North Dakota State University, Fargo, ND, USA

Received 5 July 2004; accepted 27 June 2005; published online 3 August 2005 diploid males in the vespid Euodynerus foraminatus suggests that the production of diploid males need not always represent a reproductive dead-end (Cowan and Stahlhut, 2004). However, Cowan and Stahlhut (2004) argue that even reproductively viable diploid males interfere with an ovipositing female's ability to control offspring sex ratio, and therefore represent a cost despite their reproductive viability.

CSD is known from symphytans and the clade that includes the Aculeata and Ichneumonoidea. Symphytans (sawflies) are basal within Hymenoptera, and gave rise to the Aculeate/Ichneumonoid clade and some other large hymenopteran groups (Ronquist, 1999) in which CSD appears to be lacking (Skinner and Werren, 1980; Cook, 1993b; Stouthamer and Kazmer, 1994). CSD is absent in some aculeate and ichneumonoid species as well, however. For instance, Goniozus nephantidis (Chrysidoidea: Bethylidae) is a relatively derived aculeate (Ronquist, 1999) from which both single- and multiplelocus CSD have been ruled out (Cook, 1993a). Within the Ichneumonoidea, the few known species that lack CSD are also in relatively derived subfamilies (see below). These patterns suggest that there has been evolution away from CSD to one or more other forms of sex determination that do not penalize inbreeding (Skinner and Werren, 1980; Cook, 1993a; Beukeboom, 1995; Dobson and Tanouye, 1998).

The hymenopteran family with the most information on phylogenetic patterns of sex determination is the 
Braconidae, although information remains rather sparse in this group (Wu et al, 2003). Sex determination has been investigated in members of only three of about 40 subfamilies: the relatively basal Braconinae, the derived Microgastrinae, and the intermediate Alysiinae (see Quicke and van Achterberg, 1990; Wharton et al, 1992; van Achterberg and Quicke, 1992; and Belshaw et al (1998) for detailed treatments of the relationship among braconid subfamilies). Thus far, CSD has been confirmed in the braconine genus Habrobracon, and the microgastrine Microplitis croceipes (Whiting, 1961; Steiner and Teig, 1989). CSD has been definitively ruled out in two species of alysiines, and one microgastrine (Beukeboom et al, 2000; Niyibigira et al, 2004a, b). Thus, it appears from the limited information available that CSD is present in basal braconids, but that evolution away from CSD has occurred in more derived subfamilies. Under this scenario, the presence of CSD in the Microgastrinae would indicate a long evolutionary history of CSD retention. An alternative hypothesis is that CSD can reappear in lineages after having been lost, although this is considered unlikely because of the presumed genetic load associated with diploid male production (Crozier, 1971; Bull, 1981).

Here, we determine whether single-locus CSD operates in the braconid Heterospilus prosopidis Viereck, which is in the subfamily Doryctinae, itself considered to be basal to the Braconinae, and therefore near the base of the braconid clade (Wharton et al, 1992). A previous unpublished test of CSD in $H$. prosopidis was inconclusive due to difficulties in assessing developmental mortality and the lack of a true outcross, but failed to find an increase in the frequency of male production with generations of inbreeding (Cook, 1991; J Cook, personal communication). Thus, contrary to expectations based upon phylogenetic placement of $H$. prosopidis, Cook's (1991) preliminary work suggests that CSD may be absent in this species.

\section{Materials and methods}

\section{Insects}

Heterospilus prosopidis attacks a number of seed weevils in the family Bruchidae, by depositing a single egg on the outside surface of late-stage larvae or prepupae, which themselves develop within dried beans (Charnov et al, 1981; Jones, 1982; Fujii, 1983). In our experiments, two $H$. prosopidis populations were used, one from material collected in Hawaii, USA, and the other from material collected in Arizona, USA. The two populations had been cultured separately in Japan on azuki beans, Vigna angularis (Willd.) Ohwi and Ohashi var. dainagon, infested by Callosobruchus chinensis (L.) (Shimada and Fujii, 1985; Tuda and Shimada, 1995). The Hawaiian material originated from a release of 2300 adults introduced from Texas, USA for the biological control of Algarobius prosopis (Lec.) (Coleoptera: Bruchidae) in 1910 (Clausen, 1978). A sample of $50 \mathrm{H}$. prosopidis that were presumably descendents of this population was moved to Japan in 1975 (K Fujii, personal communication). The Arizona population was collected in Arizona, USA, and moved to Japan in 1999 as a population of 400500 individuals. Both cultures were moved to the University of Minnesota in 2000 for experiments, where they were maintained as separate colonies at $25^{\circ} \mathrm{C}, 70 \%$ RH and 16L:8D.

Colonies of $H$. prosopidis were maintained on Callosobruchus maculatus (Fabricius) (Coleoptera: Bruchidae), which was reared on black-eyed beans, $V$. unguiculata Walp, at $30^{\circ} \mathrm{C}, 35-40 \% \mathrm{RH}$ and 16L:8D. Adult beetles were kept in plastic petri dishes $(100 \mathrm{~mm}$ diameter $\times$ $15 \mathrm{~mm}$ high), each with about 200 black-eyed beans and groups of $20-50$ beetles. To rear $H$. prosopidis, approximately 200 beans with between one and six 4th instar larvae each (recognizable by 'windows' in the beans indicating sites of larval development) were placed in separate dishes $(100 \mathrm{~mm}$ diameter $\times 15 \mathrm{~mm}$ high) with approximately $30 \mathrm{H}$. prosopidis. A minimum of five $H$. prosopidis dishes of both populations was maintained in this manner prior to and during the experiment.

In preparation for the experiment, beetles were allowed to lay eggs on beans for 1 day and any eggs in excess of two per bean were removed. The experiment was carried out in plastic Petri dishes $(60 \mathrm{~mm}$ diameter $\times 15 \mathrm{~mm}$ high), each containing 30 beans with 604 th instar hosts. We chose 4th instars because parasitoids of both sexes are produced from this host stage (Charnov et al, 1981; Jones, 1982; Kobayashi and Shimada, 2000; Kobayashi et al, 2003). After exposure to wasps for 4 days, beans with beetles were placed individually into glass vials $(1.5 \mathrm{~mm}$ diameter $\times 6.5 \mathrm{~mm}$ high) with a drop of honey on the inside. Beans were checked daily and the numbers of wasps of each sex that emerged, as well as the number of adult beetles that emerged, were recorded. A 4-day parasitoid exposure period was used because $H$. prosopidis tend to lay a high proportion of unfertilized (male) eggs in the early stages of oviposition (Waage, 1982) with an average fecundity of 39 at $30^{\circ} \mathrm{C}$, and most of the emerged progeny arise from parasitism during the first $96 \mathrm{~h}$ (Tuda and Shimada, 1995; Schmale et al, 2001).

\section{Mating protocol}

Under $s l$-CSD, half of the fertilized offspring are expected to be diploid males when a sex allele is shared by a diploid mother and haploid father. We designed crosses to ensure shared alleles if sl-CSD were present. Putative sex alleles are by necessity shared in matings between mothers and their haploid sons because mothers are heterozygous and pass on one or the other allele to each haploid son. Mother-son matings can be achieved in $H$. prosopidis by allowing virgin females to oviposit and then holding them at low temperatures while their sons develop. However, such matings can produce unusual sex ratios because chilling for extended periods and old age can affect mating and oviposition. For this reason, we used mother-son matings to establish isofemale lines and conducted matings between siblings from these lines. Inbred isofemale lines were initiated using mother-son crosses, which were established by allowing females to parasitize a limited number of hosts as virgins, and then keeping them alive in the refrigerator $\left(6^{\circ} \mathrm{C}\right)$ while their progeny developed to adulthood (approximately 12 days). Haploid sons were then mated to their mothers. Brother-sister matings were established by pairing brothers and sisters from within isofemale lines generated by the mother-son crosses. To ensure that haploid males were used in these crosses, we used male 
progeny from virgin females and female progeny from the same females after they had been mated. This was carried out by allowing virgin mothers of a given brother-sister cross to oviposit on a limited number of hosts prior to mating in order to produce haploid sons before allowing them to mate in order to produce daughters. For outcrosses, virgin female wasps from one population (Hawaii or Arizona) were mated with virgin males from the other population.

\section{Analyses}

If diploid males experience developmental survivorship similar to that of females, the sex ratio (proportion males) expected under $s l$-CSD is: $1-f+f / 2$, where $f$ is the proportion of eggs fertilized. If diploid males die before reaching adulthood, the expected sex ratio under $s l$-CSD becomes: $(1-f) /(1-f+f / 2)$. Thus, female-biased secondary sex ratios are not possible in shared-allele matings under sl-CSD when diploid males survive, whereas a wide range of secondary sex ratios are possible if diploid males die during development. In this latter case, progeny survivorship will also be lower (by $f / 2$ ) under $s l$-CSD than under a non-CSD system of sex determination. We therefore compared sex ratios and progeny numbers stemming from inbred and outcrossed $H$. prosopidis females.

Since $H$. prosopidis eggs are laid on hosts within beans, it was not possible to directly measure survival. Instead, we estimated juvenile survival by comparing the number of hosts offered with the number of parasitoids and live hosts produced. To correct these estimates for background host mortality, we held a set of control dishes with 60 hosts as described above but with no parasitoids and recorded the number of adult hosts emerging. The number of hosts unaccounted for by adult host emergence, adult parasitoid emergence, or background host mortality was estimated as the difference between hosts offered (60) and the mean number of hosts not emerged from the control (no parasitoid) dishes minus the sum of parasitoids and hosts emerging from each experimental dish. If all of these unaccounted-for hosts were killed by $H$. prosopidis larvae that did not complete development, the $H$. prosopidis juvenile mortality rate is $x_{i} /\left(x_{i}+a_{i}\right)$, where $x_{i}$ and $a_{i}$ are the number of hosts unacounted for, and the number of $H$. prosopidis adults, respectively, from family $i$. This is a maximum estimate of juvenile mortality since it assumes that all of the unaccountedfor hosts received an $H$. prosopidis egg, whereas stinging by $H$. prosopidis females without concomitant oviposition could have resulted in host mortality as well. Ectoparasitoids like $H$. prosopidis typically paralyze hosts prior to depositing eggs and in some cases may not deposit eggs on paralyzed hosts, which then go on to die (Godfray, 1994; Quicke, 1997). While early observations suggested that host paralysis may not have been induced by H. prosopidis (Bridwell, 1918), more recent studies are consistent with strong host paralysis at stinging. Fujii and Wai (1990) showed that the weight of $H$. prosopidis adults closely reflects the weight of their hosts at the time of stinging, despite the fact that $H$. prosopidis eggs presumably take at least 2 days to hatch, a time during which host weight can increase four-fold (Fujii and Wai, 1990). This suggests strongly that $H$. prosopidis paralyze hosts prior to oviposition, and also that hosts that were stung but not used for oviposition would succumb to paralysis.

Families with less than 10 offspring were excluded from analyses, as were families consisting of only males because they were likely laid by unmated females. We tested the effects of inbreeding on the number of adult offspring, sex ratio (prop. males), and the estimated maximum mortality with $t$-tests. We also tested whether direction of outcross (female Arizona $\times$ male Hawaii or vice versa) affected these variables. Sex ratio and mortality data were arcsine-transformed prior to analysis, but actual proportions are presented. Finally, we tested whether the frequency of all-male families differed between shared-sex-allele crosses vs outcrosses using contingency tables and the likelihood-ratio $\chi^{2}$ statistic.

\section{Results}

Neither the number of adult offspring nor the estimated maximum mortality differed significantly between inbred crosses and outcrosses (Table 1). Sex ratios were significantly lower (ie, more female-biased) for inbred crosses than for outcrosses (Table 1). A higher proportion of inbred families were all-male than outbred families (17 vs 5\%; likelihood-ratio $\chi_{1,130}^{2}=4.2 ; P<0.05$ ), but all-male families only occurred in families with less than 10 offspring. For the outcrosses, the direction of the cross had no significant effect on progeny number $(t=-0.3$; $P>0.50)$, sex ratio $(t=-1.8 ; P>0.05)$, or estimated parasitoid developmental mortality $(t=0.5 ; P>0.50)$.

A more female-biased sex ratio and similar juvenile mortality in inbred crosses vs outcrosses is not consistent with $s l$-CSD. However, sl-CSD cannot be ruled out from the above analyses alone because of the possibility that both of our strains of $H$. prosopidis harbored the same two sex alleles. If this were the case, we would expect no CSD-caused difference between the sex ratios and parasitoid mortality from the inbred and outcrossed females. Three scenarios could produce this outcome: (1)

Table 1 Mean \pm SEM (standard error of the mean) progeny numbers, sex ratios and estimated maximum juvenile mortality produced by female $H$. prosopidis that were either inbred to brothers from isofemale lines, or outcrossed to males from a different population

\begin{tabular}{lcccc}
\hline Cross & Number of families & Number of progeny \pm SEM & Sex ratio (prop. males) \pm SEM & Estimated maximum juvenile mortality \pm SEM \\
\hline Inbred & 53 & $21.5 \pm 1.3$ & $0.21 \pm 0.015$ & $0.27 \pm 0.023$ \\
Outcrossed & 24 & $16.8 \pm 2.0$ & $0.30 \pm 0.035$ & $0.33 \pm 0.021$ \\
Student's $t$ & 1.95 & $>0.05$ & $<0.01$ & $-1.5^{\mathrm{a}}$ \\
$P$ & & & $-2.8^{\mathrm{a}}$ & $>0.10$ \\
\hline
\end{tabular}

${ }^{a}$ Analysis carried out on arcsine-transformed proportions. 
contamination of one of the strains along with loss of the strain receiving the contamination, (2) very low global sex allele diversity, or (3) a common ancestor to both the Hawaiian and Arizona $H$. prosopidis strains such that sex alleles were shared between the strains.

We can determine whether our sex ratio data and parasitoid mortality estimates are consistent with $s l$-CSD or whether sl-CSD can be ruled out. Depending upon the diploid male survivorship rate, certain combinations of sex ratio and parasitoid mortality are not possible under sl-CSD (Cook, 1993a). If diploid male survival is equal to that of females, female-biased secondary sex ratios are not possible in shared-sex-allele matings, regardless of
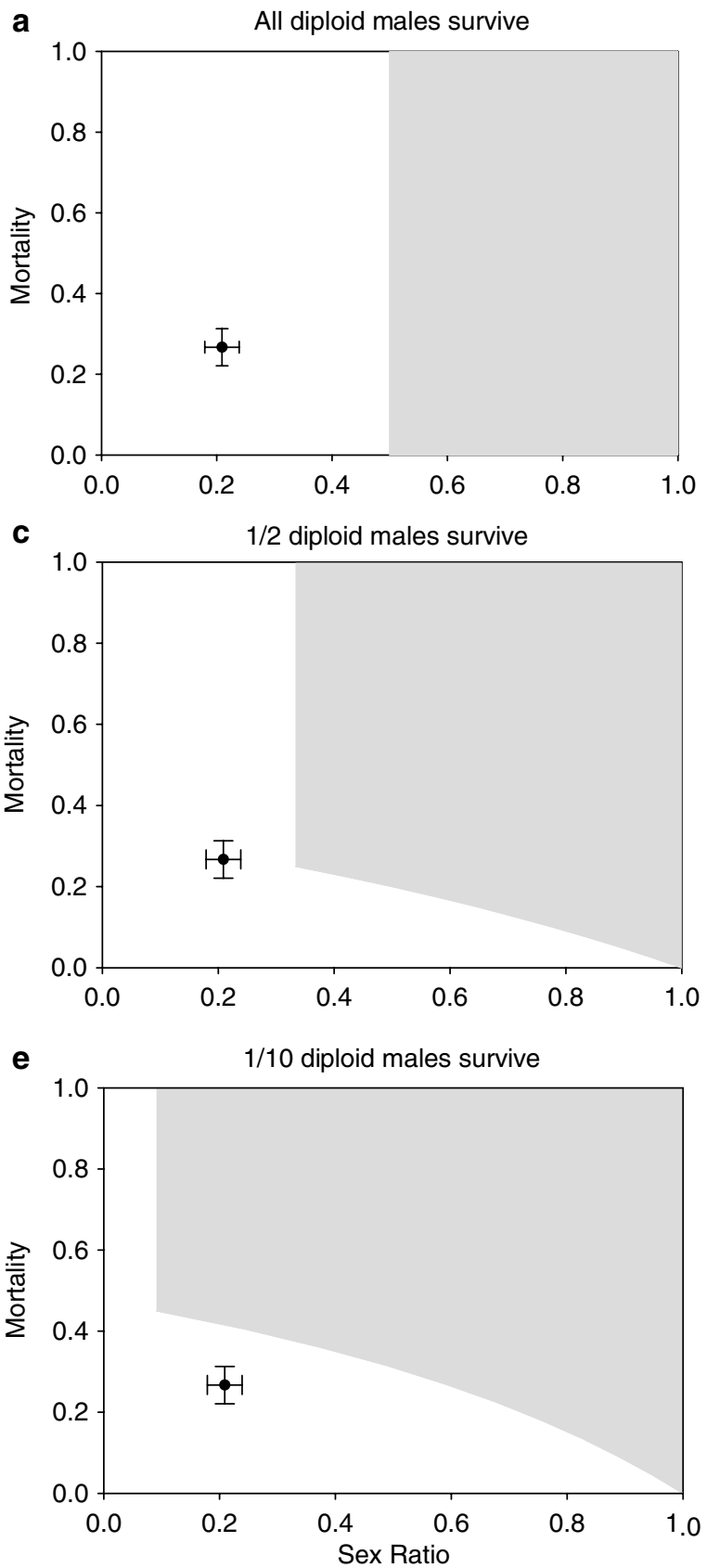

the fertilization rate. Diploid male survivorship may be reduced, however, and in this case, female-biased sex ratios are possible and are an increasing function of the fertilization rate, as discussed in the introduction. However, a minimum level of $f / 2$ developmental mortality is expected under these conditions, so that threshold levels of developmental mortality are required for any given level of secondary sex ratio to be consistent with CSD. These relationships are captured by the expression for secondary sex ratio $(r)$ as a function of the fertilization rate $(f)$ and the rate of diploid male survival $(\delta)$ under sl-CSD (Cook, 1993a; Heimpel et al, 1999; Antolin et al, 2003): $r=[1-f(1-0.5 \delta)] /[1-0.5 f(1-\delta)]$.
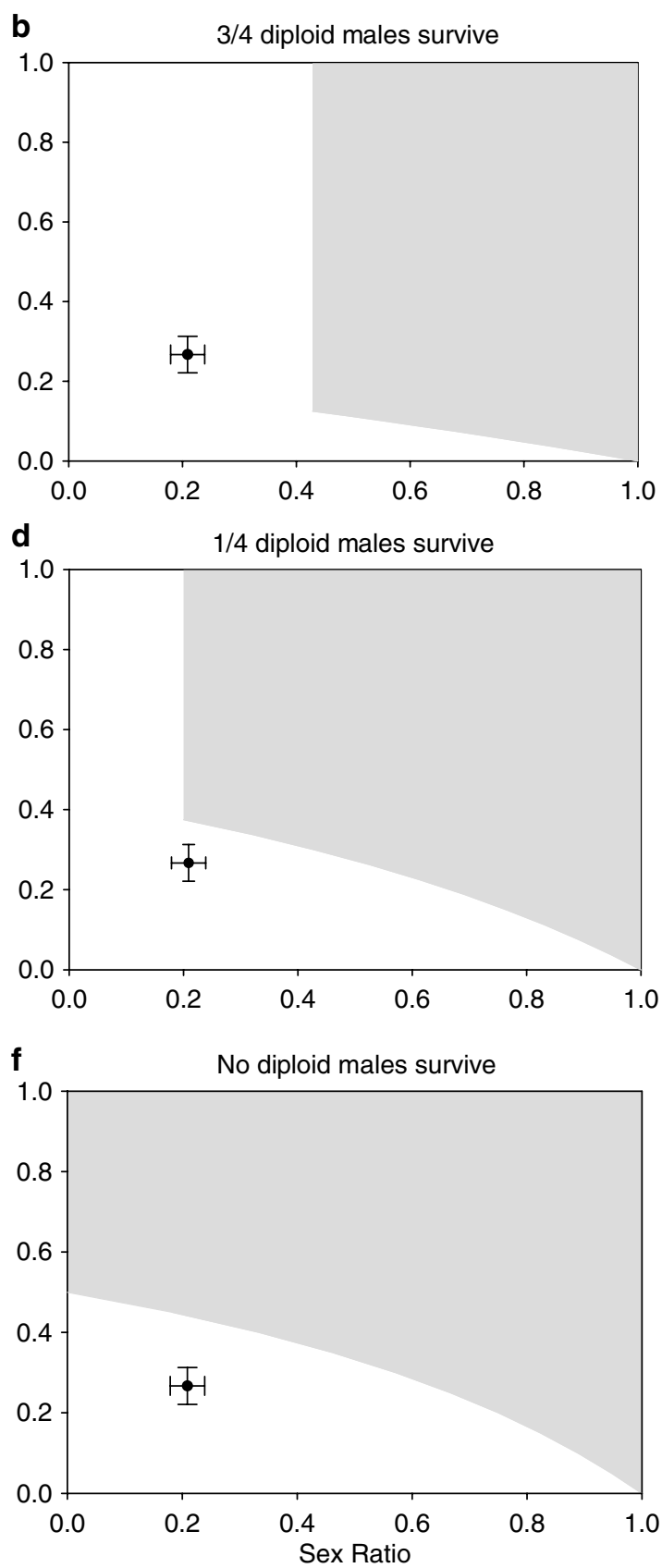

Figure 1 Combinations of developmental mortality and secondary sex ratio that are consistent with sl-CSD under six levels of diploid males survivorship. Gray denotes the parameter space that is consistent with $s l$-CSD over all possible fertilization rates. The data point represents the mean $\pm 95 \%$ confidence intervals for secondary sex ratio and estimated developmental survival from the inbred data set (see Table 1). The data are inconsistent with $s l$-CSD when they lay outside of the gray parameter space. 
We used this function to determine whether the sex ratio and mortality levels produced in our study could be consistent with CSD, or whether they could exclude CSD as a sex determining mechanism. We found no values of $f$ or $\delta$ that were consistent with CSD, as is illustrated in Figure 1 . The estimated values of sex ratio and mortality used in Figure 1 are from inbred lines only and thus represent crosses that should exhibit characteristics of CSD, if indeed it operated in this species. However, the sex ratios produced by our $H$. prosopidis are too femalebiased to be consistent with CSD showing high survival of diploid males (Figure 1a-c). Additionally, the estimated mortality rates are too low to explain the level of female bias under a low diploid-male survival model (Figure 1d-f). Thus, we can rule out sl-CSD under all levels of diploid male survivorship, and conclude that $s$ CSD does not operate in $H$. prosopidis. The sex ratio and mortality values produced in our data set came closest to being consistent with sl-CSD at intermediate levels of hypothetical diploid male survivorship. This is because high levels of diploid male survivorship lead to sex ratios that are much higher (ie, more male-biased) than we observed, and low levels of diploid male survivorship are associated with much higher juvenile mortality than we observed (Figure 1).

\section{Discussion}

Lack of $s l$-CSD in $H$. prosopidis brings the number of species without CSD in the family Braconidae to five, and the number of subfamilies containing at least one species without CSD to three (Beukeboom et al, 2000; Niyibigira et al, 2004a,b). More importantly, our finding represents the most basal phylogenetic placement of a mode of sex determination other than $s l$-CSD in the family Braconidae. The Doryctinae (to which $H$. prosopidis belongs) is considered basal to the Braconinae (van Achterberg, 1984; Quicke and van Achterberg, 1990; Wharton et al, 1992; Belshaw et al, 1998), where CSD was discovered in various species of Habrobracon (Whiting, 1961; Holloway et al, 1999; Weiser et al, 2004).

The pattern of CSD that is beginning to emerge in the Braconidae could be explained by one of two hypotheses. First, it is possible that CSD is not the ancestral sex determination mechanism in the family. The genetic load associated with the production of inviable or sterile diploid males makes multiple origins of CSD seem unlikely (Crozier, 1971; Bull, 1981), but it may be that the ancestral status of CSD needs to be reconsidered. In particular, the recent finding of functionally reproductive diploid males in a vespid provides an evolutionary mechanism by which the disadvantages associated with diploid male production can be greatly attenuated (Cowan and Stahlhut, 2004; Stahlhut and Cowan, 2004). Second, the mode of sex determination may be very evolutionary labile in the Braconidae (and perhaps other taxa) making broad-scale phylogeny a poor predictor of sex determination mechanism. This hypothesis is supported in general terms by a recognition that sex determination is more labile than previously assumed in both invertebrates and vertebrates (Cook, 2002; Kraak and Pen, 2002). There is also reason to suspect that a single genus (Cotesia Cameron, within the Microgastrinae) may contain species that have and do not have CSD.
On the one hand, Niyibigira et al (2004b) have excluded CSD from C. flavipes Cameron, and field studies suggest no CSD in C. sesamiae (Cameron) as well (Niyibigira et al, 2004a). On the other hand, there is an unpublished report of diploid males being produced by inbred C. rubecula (Marshall) (Stouthamer et al, 1992; W Steiner, personal communication), and patterns of inbreeding and sex ratio suggest CSD in C. glomerata (Gu and Dorn, 2003).

The question of what the alternatives are to CSD remains unresolved in the Braconidae, although Dobson and Tanouye (1998) have presented evidence consistent with a genomic imprinting model of haplo-diploidy for the pteromalid Nasonia vitripennis (Walker) in which unfertilized eggs develop as males because they are never exposed to gene products produced by the male genome. Other alternative models of sex determination that could explain haplo-diploidy in the Hymenoptera include (i) a genic balance mechanism in which the effects of female-determining genes outweigh effects of male-determining genes in diploids, (ii) a maternal effects model in which sex is determined by the ratio of nuclear to cytoplasmically inherited gene products, and (iii) multiple-locus CSD ( $m l$-CSD), in which hemizygosity leads to haploid males as in sl-CSD, but diploid males are only produced in individuals homozygous at two or more sex loci (reviewed by Beukeboom, 1995). We attempted a test of $m l-C S D$ as part of the research reported here, but the results were inconclusive so they are not reported here. $m l-C S D$ has not been ruled out in many of the cases of confirmed CSD, but it has been ruled out for a number of species with sl-CSD and in species with mechanisms of sex determination other than CSD (Wu et al, 2003).

Our understanding of sex determination in the Hymenoptera has expanded over the last decade with increasing recognition that CSD is not the only sex determination mechanism in the Ichneumonoidea, and that the mechanisms for achieving haplo-diploidy may be subject to relatively rapid evolution. Further investigations into alternative models of sex determination in Hymenoptera (Dobson and Tanouye, 1998), reduction in the costs associated with diploid males (Cowan and Stahlhut, 2004), as well as discoveries of the genetic mechanisms of CSD itself (Beye et al, 2003; Hasselmann and Beye, 2004) will complement elucidation of phylogenetic patterns of sex determination to provide a more comprehensive picture of the evolution of Hymenopteran haplo-diploidy.

\section{Acknowledgements}

We thank Frank J Messina for supplying beetle hosts, and M Shimada and A Kobayashi for their Arizona strain of $H$. prosopidis. We also thank Jeff Berg, Seth Bomgren, Randy Hedlund and Markeeta Keyes for rearing assistance, Jetske De Boer and two anonymous reviewers for advice on the manuscript, J Cook for discussing his unpublished work on $H$. prosopidis with us, and K Fujii and $W$ Steiner for sharing unpublished information. This study was supported by United States Department of Agriculture National Research Initiative Grant 0002995 to $\mathrm{KRH}, \mathrm{GEH}, \mathrm{PJO}$ and RWF, and by the University of Minnesota Agricultural Experiment Station. 


\section{References}

Antolin MF, Ode PJ, Heimpel GE, O'Hara RB, Strand MR (2003). Population structure, mating system, and sex determining allele diversity of the parasitoid wasp Habrobracon hebetor. Heredity 91: 373-381.

Belshaw R, Fitton M, Herniou E, Gimeno C, Quicke DLJ (1998). A phylogenetic reconstruction of the Ichneumonoidea (Hymenoptera) based on the D2 variable region of $28 \mathrm{~S}$ ribosomal DNA. Systematic Entomology 23.

Beukeboom LW (1995). Sex determination in Hymenoptera - a need for genetic and molecular studies. BioEsseys 17: 813-817.

Beukeboom LW, Ellers J, van Alphen JJM (2000). Absence of single-locus complementary sex determination in the braconid wasps Asobara tabida and Alysia manducator. Heredity 84: 29-36.

Beye M, Hasselmann M, Fondrk MK, Page Jr RE, Omholt SW (2003). The gene csd is the primary signal for sexual development in the honeybee and encodes an SR-type protein. Cell 114: 419-429.

Bridwell JC (1918). Notes on the Bruchidae and their parasites in the Hawaiian Islands. Proc Hawaiian Entomol Soc 3: 465-505.

Bull JJ (1981). Coevolution of haploidiploidy and sex determination in the Hymenoptera. Evolution 35: 568-580.

Charnov EL, Los-den Hartogh RL, Jones WT, van den Assem J (1981). Sex ratio evolution in a variable environment. Nature 289: 27-33.

Clausen CP (1978). Introduced Parasites and Predators of Arthropod Pests and Weeds: a World Review. United States Department of Agriculture, Agriculture Research Service: Washington, DC, USA, 545 pp.

Cook JM (1991). Sex determination and sex ratios in parasitoid wasps. PhD Thesis, University of London.

Cook JM (1993a). Experimental tests of sex determination Goniozus nephantidis (Hymenoptera: Bethylidae). Heredity 71: 130-137.

Cook JM (1993b). Sex determination in Hymenoptera: a review of models and evidence. Heredity 71: 421-435.

Cook JM (2002). Sex determination in invertebrates. In: Hardy ICW (ed) Sex Ratios: Concepts and Research Methods. Cambridge University Press: Cambridge, pp 178-194.

Cook JM, Crozier RH (1995). Sex determination and population biology in the Hymenoptera. Trend Ecol Evol 10: 281-286.

Cowan DP, Stahlhut JK (2004). Functionally reproductive diploid and haploid males in an inbreeding hymenopteran with complementary sex determination. Proc Natl Acad Sci USA 101: 10374-10379.

Crozier RH (1971). Heterozygosity and sex determination in haploidiploidy. Am Nat 105: 399-412.

Dobson SL, Tanouye MA (1998). Evidence for a genomic imprinting sex determination mechanism in Nasonia vitripennis (Hymenoptera: Chalcidoidea). Genetics 149: 233-242.

Fujii K (1983). Resource dependant stability in an experimental laboratory resource - herbivore-carnivore system. Res Popul Ecol Suppl 3: 15-26.

Fujii K, Wai KM (1990). Sex-ratio determination in three wasp species ectoparastic on bean weevil larvae. In: Fujii K, Gatehouse AMR, Johnson CD, Mitchel R, Yoshida T (eds) Bruchids and Legumes: Economics, Ecology and Coevolution. Kluwer: Dordrecht, The Netherlands, pp 331-340.

Godfray HCJ (1994). Parasitoids: Behavioral and Evolutionary Ecology. Princeton University Press: Princeton, NJ, USA.

$\mathrm{Gu} \mathrm{H}$, Dorn S (2003). Mating system and sex allocation in the gregarious parasitoid Cotesia glomerata. Anim Behav 66: 259-264.

Hasselmann M, Beye M (2004). Signatures of selection among sex-determining alleles of the honey bee. Proc Nat Acad Sci USA 101: 4888-4893.

Heimpel GE, Antolin MF, Strand MR (1999). Diversity of sexdetermining alleles in Bracon hebetor. Heredity 82: 282-291.
Holloway A, Heimpel GE, Strand MR, Antolin MF (1999). Survival of diploid males in Bracon sp. near hebetor (Hymenoptera: Braconidae). Ann Entomol Soc Amer 92: 110-116.

Jones WT (1982). Sex ratio and host size in a parasitoid wasp. Behav Ecol Sociobiol 10: 207-210.

Kobayashi A, Shimada M (2000). Field sex ratio of a braconid parasitoid wasp, Heterospilus prosopidis (Hymenoptera: Braconidae), in the Southwestern United States: concordance with host-quality model. Ann Entomol Soc Am 93: 819-824.

Kobayashi A, Tanaka Y, Shimada M (2003). Genetic variation of sex allocation in the parasitoid wasp Heterospilus prosopidis. Evolution 57: 2659-2664.

Kraak SBM, Pen I (2002). Sex-determining mechanisms in vertebrates. In: Hardy ICW (ed) Sex ratios: concepts and research methods. Cambridge University Press: Cambridge, pp 158-177.

Niyibigira EI, Overholt WA, Stouthamer R (2004a). Cotesia flavipes Cameron and Cotesia sesamiae (Cameron) (Hymenoptera: Braconidae) do not exhibit complementary sex determination: evidence from field populations. Appl Entomol Zool 39: 705-715.

Niyibigira EI, Overholt WA, Stouthamer R (2004b). Cotesia flavipes Cameron (Hymenoptera: Braconidae) does not exhibit complementary sex determination (ii) evidence from laboratory experiments. Appl Entomol Zool 39: 717-725.

Quicke DLJ (1997). Parasitic Wasps. Chapman \& Hall: London, UK.

Quicke DLJ, van Achterberg K (1990). Phylogeny of the subfamilies of the family Braconidae (Hymenoptera: Ichneumonidae). Zool Verh Leiden 258: 1-95.

Ronquist F (1999). Phylogeny of the Hymenoptera (Insecta): the state of the art. Zool Scripta 28: 3-11.

Schmale I, Waeckers FL, Cardona C, Dorn S (2001). Control potential of three hymenopteran parasitoid species against the bean weevil in stored beans: the effect of adult parasitoid nutrition on longevity and progeny production. Biol Control 21: 134-139.

Shimada M, Fujii K (1985). Niche modification and stability of competitive systems. I. Niche modification process. Res Popul Ecol 27: 185-201.

Skinner SW, Werren JH (1980). The genetics of sex determination in Nasonia vitripennis (Hymenoptera, Pteromalidae) Genetics 94: s98.

Stahlhut JK, Cowan DP (2004). Single-locus complementary sex determination in the inbreeding wasp Euodynerus foraminatus Saussure (Hymenoptera: Vespidae). Heredity 92: 189-196.

Steiner WWM, Teig DA (1989). Microplitis croceipes: genetic characterization and developing insecticide resistant biotype. Southwest Entomol 12: 71-87.

Stouthamer R, Kazmer DJ (1994). Cytogenetics of microbeassociated parthenogenesis and its consequences for gene flow in Trichogramma wasps. Heredity 73: 317-327.

Stouthamer R, Luck RF, Werren JH (1992). Genetics of sex determination and improvement of biological control using parasitoids. Environ Entomol 21: 427-435.

Tuda M, Shimada M (1995). Developmental schedules and persistence of experimental host-parasitoid systems at two different temperatures. Oecologia 103: 283-291.

van Achterberg C (1984). Essay of the phylogeny of Braconidae (Hymenoptera: Ichneumonoidea). Ent Tidskr 105: 41-58.

van Achterberg K, Quicke DLJ (1992). Phylogeny of the subfamilies of the family Braconidae: a reassessment assessed. Cladistics 8: 237-264.

Waage JK (1982). Sex ratio and population dynamics in natural enemies - some possible interactions. Ann Appl Biol 101: 159-164.

Weiser L, Antolin MF, Wu Z, Heimpel GE (2004). Does temperature affect diploid male production in Habrobracon hebetor (Say) (Hymenoptera: Braconidae)? J Hymen Res 13 103-109. 
Werren JH (1993). The evolution of inbreeding in haplodiploid organisms. In: Thornhill NW (ed) The Natural History of Inbreeding and Outbreeding. University of Chicago Press: Chicago, IL, pp 42-59.

Wharton RA, Shaw SR, Sharkey MJ, Wahl DB, Woolley JB, Whitfield JB, Marsh PM, Johnson W (1992). Phylogeny of the subfamilies of the family Braconidae (Hymenoptera: Ichneumonoidea): a reassessment. Cladistics 8: 199-235.
Whiting AR (1961). Genetics of Habrobracon. Adv Genet 10: 295-348.

Whiting PW (1943). Multiple alleles in complementary sex determination of Habrobracon. Genetics 24: 110-111.

Wu Z, Hopper KR, Ode PJ, Fuester RW, Chen J, Heimpel GE (2003). Complementary sex determination in hymenopteran parasitoids and its implications for biological control. Entomol Sinica 10: 81-93. 